\title{
10 Kritische Theorie und Psychoanalyse. Die Spur Freuds
}

\subsection{Präsenz und verdeckte Spur der Psychoanalyse}

Die Präsenz der Psychoanalyse in der Dialektik der Aufklärung ist nicht einfach zu verorten. Sie ist kein zentrales, explizites Thema, und doch durchdringt sie die Schrift als ganze. Dies entspricht der Auseinandersetzung mit ihr im Gesamtwerk von Horkheimer und Adorno. Beide Autoren befassen sich schon früh und in unterschiedlichen Phasen ihres Schaffens mit Freud, ohne dass dieser - wie etwa andere Theoretiker oder Komponisten bei Adorno - Gegenstand einer systematischen Abhandlung geworden wäre. Ähnlich bildet die Psychoanalyse für die Kritische Theorie insgesamt eine durchgehende Referenzposition, deren Stellung sich im Laufe der Jahrzehnte allerdings stark verändert und nach Autoren variiert (vgl. Bonß 1982; Dahmer 2012; Lohmann 2006). Für Horkheimer und Adorno steht sie seit den zwanziger und dreißiger Jahren im Blick. Adorno hat sich bereits in der (zurückgezogenen) Habilitationsschrift von 1927 mit Freuds Theorie des Unbewussten im Kontext der Transzendentalphilosophie und philosophischen Ideengeschichte beschäftigt (AGS 1, 79-322); Horkheimer hat der Psychologie eine wichtige Stellung im Konzept eines interdisziplinären Materialismus eingeräumt und die Psychoanalyse zu einem Pfeiler der Kritischen Theorie gemacht (HGS 3, 48-69). Zugleich setzen sich beide Autoren kritisch von bestimmten („revisionistischen“) Strömungen der psychoanalytischen Tradition (Karen Horney, Erich Fromm) ab, gegen welche sie zentrale Konzepte der originären Freudschen Theorie (u.a. der Triebpsychologie) stark machen; teils opponieren sie gegen herrschende Praxisformen der analytischen Therapie, teils gegen Zweideutigkeiten der Freudschen Terminologie selbst. Die im Laufe der Zeit changierende Doppelseitigkeit von Aneignung und Kritik prägt auch das psychoanalytische Grundgerüst der Dialektik der Aufklärung. Es soll im Folgenden anhand leitender Fragestellungen und thematischer Schwerpunkte konkreter entfaltet und in seinem Stellenwert verdeutlicht werden. 


\subsection{Kritische Gesellschaftstheorie und Psychoanalyse}

Auszugehen ist von der Frage, welches Anlass und Grund für die Berücksichtigung der Freudschen Theorie in einer marxistisch inspirierten kritischen Gesellschaftstheorie sind. Dass sich eine konzeptuelle Verschränkung beider Forschungsperspektiven nicht von selbst versteht, gilt nicht nur im Blick auf den Gegensatz von revolutionärer Hoffnung und anthropologisch-kulturtheoretischem Pessimismus (Jay 1976, 113-142), sondern auch auf das divergierende Theorieinteresse soziologischer und psychologischer Analyse. Motiviert ist die Verbindung durch eine Irritation in der Durchführung des gesellschaftskritischen Programms, das auf die Widerstandskraft des unterdrückten Lebens und die Überzeugungskraft rationaler Kritik setzte; provoziert wird sie durch die Irrationalität eines gegen sein Eigeninteresse gerichteten Verhaltens, die Selbstwidersprüchlichkeit eines in sich rückläufigen, selbstdestruktiven Fortschritts. Der Titel einer Dialektik der Aufklärung steht exemplarisch für die Figur einer sich selbst zerstörenden Vernunft, welche das Projekt menschlicher Emanzipation in ihr Gegenteil verkehrt. Psychoanalyse wendet sich jener Tiefenschicht im Seelenleben der Subjekte zu, welche die Schwierigkeit, ja, Selbstbehinderung freier Selbstwerdung bedingt. Ihr Gegenstand sind Pathologien, die nicht nur irgendwelche Dysfunktionen des Verhaltens, sondern Probleme des Selbstseins betreffen. Die beiden Figuren der kulturgeschichtlichen Rückläufigkeit und der persönlichen Selbstbehinderung stehen in offenkundiger Korrespondenz. Zugleich besitzen sie - und darin liegt die Pointe ihrer Verschränkung - wechselseitig explikative Funktion. Auf der einen Seite ist es unmöglich, die geschichtlich-gesellschaftlichen Verhältnisse, im Besonderen das Funktionieren der sozialen Repression und die Selbstverkehrung aufklärerischer Emanzipation, unabhängig von den psychischen Mechanismen, über welche sie vermittelt sind, aufzuhellen: Darin wendet sich eine interdisziplinäre Kritische Theorie gegen Ansätze eines orthodoxen Marxismus oder eine platte Soziologisierung; weder ökonomische Gesetze noch politische Herrschaft oder die Logik der Klassenkampfes bieten hier zulängliche Explikationsmuster. $\mathrm{Zu}$ begreifen ist, wie die Gesellschaft in die Seele hineinwirkt und über diese ihre eigene Herrschaft durchsetzt. Diese Interaktion, die sich einer kritischen Gesellschaftsanalyse generell aufdrängt, verschärft sich im Zeitalter der Totalitarismen. Auf der anderen Seite sind die Formen des Seelenlebens und das Handeln der Individuen nicht aus sich heraus, losgelöst von sozialen und realhistorischen Bedingungen, erklärbar. Die wechselseitige Verweisung zwischen den heterogenen Fragerichtungen der Psychoanalyse und der Kritik der politischen Ökonomie gehört zur These und zum epistemologischen 
Anspruch des auch für die Dialektik der Aufklärung verbindlichen Grundkonzepts kritischer Theorie.

Wenn der erste Leitbegriff der psychoanalytischen Deutung der Begriff des Unbewussten ist, so findet in der umrissenen Verschränkung eine doppelte Radikalisierung des mit diesem Begriff Anvisierten statt: eine Radikalisierung vom Unbewußten zum Irrationalen und eine Vertiefung von der individuell-bewusstseinsphilosophischen zur sozialen und historischen Genese.

Als solches steht das Unbewusste für eine strukturelle und genetische Tiefenschicht, die in der klassischen Bewusstseinsphilosophie wie der Geschichtsund Kulturphilosophie nicht thematisch wird; es repräsentiert das dem subjektiven Tun Vorausliegende und seinem Zugriff sich Entziehende. Verwicklungen der Menschheitsgeschichte wie Inkonsistenzen menschlichen Handelns können ohne Aufhellung dieser Schicht nicht verstanden oder gar vernünftig korrigiert werden. Das Unbewusste markiert eine Grenze des subjektiven Sicherkennens und freien Über-sich-Verfügens, ähnlich wie solche Grenzen auch durch die soziale und historische Verwurzelung oder die leibliche Verfassung des Subjekts gesetzt sind. Indessen geht es der Psychoanalyse um mehr als eine defizitäre Selbsttransparenz, eine nicht-abgelegte Naturverhaftung und verbleibende Selbstfremdheit. Das Unbewusste ist nicht nur die Zone des Unaufgehellten, der nicht einholbare blinde Fleck im Selbstsein, wie er auch von einer transzendentalphilosophischen Subjektphilosophie reflektiert werden kann. Es steht nach psychoanalytischer Lesart für ein unbewusst Gemachtes, ein Verdrängtes, das aufgrund seiner Konfliktualität oder Negativität nicht bewusst werden kann und darf. Uneinholbar ist dem Bewusstsein nicht nur das Fremde und Verdeckte, sondern in anderer Hinsicht das Unerträgliche, das abgewehrte Negative und dasjenige, was sich von sich aus dem Begreifen widersetzt, das Widersinnige und Irrationale. Es ist eine Dimension des Erlebens, die in der Urgeschichte des Subjekts, im Schicksal seines Trieblebens und in der sozialen Konstellation seiner Selbstwerdung verankert ist und deren Kräfte und Konflikte sich nach Horkheimer und Adorno in der Selbstverkehrung der geschichtlichen Entwicklung auswirken. Die Gegenläufigkeit fortschreitender Aufklärung ist keine immanente Wendung der kulturellen Evolution.

Allerdings, so die komplementäre Grundthese, ist dieser dem Bewusstsein entzogene Bereich nicht allein im Seelenleben und in der Konstitution des Individuums festzumachen. Kritische Theorie distanziert sich vom „monadologischen“ Charakter des Freudschen Unbewussten (HGS 12, 441), nicht um stattdessen auf ein kollektives Unbewusstes zu setzen, sondern um die soziale und historische Vermitteltheit des Bewusstseins herauszustellen. Dabei geht es um eine genetische Betrachtung, die hinter die Archäologie des Bewusstseins auf die Urgeschichte des Menschen zurückgreift. Gerade mit Bezug auf die Pathologien 
der Gegenwart, die Deformationen des angepassten Subjekts der Massenkultur, sind die Ursachen jenseits der innerpsychischen Konflikte und psychoanalytisch diagnostizierten Neurosen nach Adorno in „noch früheren Phasen der Kindheitsentwicklung“, in einem „gleichsam prähistorischen Eingriff“ zu suchen (Adorno 1951, 69-70). Die herrschende Psychoanalyse, so der kritische Einwand, ist kaum in der Lage, die Normalität und verordnete Fröhlichkeit der beschädigten Gesellschaft in ihrem abgründigen Zwang zu erfassen (Adorno 1951, 69-70). Wenn das Schicksal des Menschen, auch in seinem affirmativen Wollen und seiner Sehnsucht, auf eine „Urgeschichte“ des Subjekts zurückweist $(70-71,78,189)$, so gilt Analoges für die Destruktivität des Handelns und die Selbstauflösung der Ratio, wie sie exemplarisch in einer „Urgeschichte des Antisemitismus“ (7) zum Tragen kommt, welche nicht wie bei Freud als psychologische Genese, sondern in archaischen, aber gesellschaftlich realen Bewegungen nachzuzeichnen ist (HGS 16, 764.). Diese Geschichte in ihren Wurzeln und ihren Manifestationen zu entfalten, ist das Projekt der Dialektik der Aufklärung.

Damit ist die allgemeine Verschränkung zwischen den Fragehorizonten der Psychoanalyse einerseits, der Gesellschafts- und Kulturkritik andererseits umrissen. Inhaltlich korrespondiert ihr die Verschränkung zwischen der Genese und Seinsweise des Subjekts einerseits, der Menschheits- und Kulturgeschichte andererseits. Im Folgenden sollen einzelne Knotenpunkte dieser Konstellation näher vergegenwärtigt und als Interpretament der Dialektik der Aufklärung expliziert werden.

\subsection{Trieb, Triebunterdrückung, Entsagung}

Der Mensch versteht sich nicht aus sich heraus: aus der bewussten Selbstbeziehung des Subjekts, dem rationalen Denken, der geistigen Natur des Selbst. Menschliches Sein hat seinen Grund in einem Anderen, das dem bewussten Tun und Überlegen voraus und zugrunde liegt. $\mathrm{Zu}$ den zentralen Thesen und kulturgeschichtlichen Revolutionen der Psychoanalyse gehört die Rehabilitierung der Triebnatur des Menschen gegen ihre Marginalisierung im leitenden Menschenbild wie ihre Unterdrückung in der herrschenden Kultur. Was seit der platonisch-aristotelischen Gliederung der Seelenteile als Herrschaft der Vernunft über die begehrenden Leidenschaften postuliert wurde, wird hier als Makel, jedenfalls als kritischer Punkt in der historischen Konstitution des Selbst herausgestellt. Es ist eine Unterdrückung, die mit Verlusten einhergeht, welche sich in Beschädigungen des individuellen wie des sozialen Lebens äußern. Erfordert ist eine Einsicht in die anthropologisch fundierende Rolle der Triebnatur ebenso wie die realhistorischen Konstellationen ihrer Disziplinierung. 
Gegen die revisionistische Tendenz zur Entsexualisierung beharren Horkheimer und Adorno auf einer gewissen orthodoxen Lektüre der Psychoanalyse, welche die frühe Libidotheorie wie die spätere Todestriebhypothese ernst nimmt und in der Zeitdiagnose und Kulturkritik zum Tragen bringt. Explizit wendet sich Adorno gegen die in der „revidierten Psychoanalyse“ von Erich Fromm und Karen Horney praktizierte „Polemik gegen Freuds Triebpsychologie“ und die Abwehr der schreckhaften Phänomene wie der Kastrationsdrohung, durch welche der kritische „Stachel der psychologischen Erkenntnis“ verloren geht (AGS 8, 20, 23, 25 26). Die Einseitigkeit der philosophischen Begriffsgeschichte des Unbewussten (AGS 1, 79-322) liegt eben in Vernachlässigung des materialistischen Moments der Organlust zugunsten der rein erkenntnistheoretischen Fragestellung (vgl. Rantis 2001, 79). Dabei gilt der Akzent nicht primär dem materialistisch-naturalistischen Beschreibungsansatz als solchem als vielmehr dem Rückgang in die Tiefe des Seelenlebens als Grund der destruktiven wie der affirmativen Lebenstendenzen. Nicht zuletzt die Befreiung der reinen körperlichen Lust, die stellvertretend für die Utopie des zweckfreien Glücks steht, kommt erst jenseits der „bürgerlichen Verachtung des Triebs“ in den Blick (AGS 4, 65-72). In der Kirke-Episode der Odyssee wird in der magischen Verwandlung in die naturale Seinsweise zugleich mit dem ältesten Sinn des Geruchs die darin verborgene „Spur der Lust“ evoziert (78). Der Trieb ist Grund und emanzipatorisches Potential.

Umso dramatischer nimmt sich seine kategorische Unterdrückung aus. Wenn Freud die Repression des Triebhaften als Bedingung der normalen Selbstwerdung und Moment der kulturellen Sublimation zeichnet, so nimmt diese Zurückdrängung in der menschheitsgeschichtlichen Optik der Dialektik der Aufklärung Züge der Gewalt und Zerstörung an. „Furchtbares hat die Menschheit sich antun müssen, bis das Selbst, der identische, zweckgerichtete, männliche Charakter des Menschen geschaffen war“ (40). Der Triebverzicht, das Verschmähen der Lust wird zur Selbstverstümmelung als Bedingung der Selbst- und Naturbeherrschung (80). Das den Gottheiten zur Zähmung der Natur dargebrachte Opfer richtet sich gegen das Selbst; die Verinnerlichung der gehemmten Aggression und die Introversion des Opfers werden zu Scharnieren der Zivilisation, die durch externe Mächte erzwungene Versagung vertieft sich zur Entsagung (62). Die reflexiv gewendete Unterdrückung des Selbst verschmilzt mit der sozialen Herrschaft der Mächtigen, wird als deren Effekt zu ihrem Medium. Die Beherrschung des Triebs, die für das klassische Menschenbild die Basis innerer Souveränität und Selbstaffirmation darstellt, wird zum Hebel der Selbstverleugnung und Vernichtung des Selbst. Die Beherrschung verstärkt sich zur Lustfeindschaft und destruktiven Abwehr. So liegt es nahe, dass in einer bestimmten Verbindung von Freud und Marx die befreite Sinnlichkeit umgekehrt zur eigentlichen revolutionären Kraft mutieren kann (Marcuse 1969). 


\subsection{Naturbeherrschung, Mimesis, Eingedenken der Natur}

Was die Psychoanalyse mit Bezug auf den Triebgrund des Lebens reflektiert, wird in der Urgeschichte der Subjektivität als Naturverhältnis zum Thema. Auch hier steht ein Selbstverhältnis in Frage, und auch hier findet nach der Dialektik der Aufklärung eine analoge Entfremdung statt, die zwischen Naturunterwerfung, Naturvergessenheit und Naturzerstörung oszilliert. Wenn im klassischen Entwicklungsschema bei Hegel das Zusichkommen des Geistes ein Herausgehen aus dem Naturzustand und Überwinden des naturalen Außersichseins beinhaltet, so ist diese aneignende Überwindung im zivilisatorischen Prozess zu einer dissoziierenden Repression geworden. Sie manifestiert sich nicht nur im äußeren Naturverhältnis, sondern ebenso in der Tabuisierung der eigenen Natur, der „Haßliebe gegen den Körper“, die den Leib zum Ding degradiert; „in der Selbsterniedrigung des Menschen zum corpus rächt sich die Natur dafür, daß der Mensch sie zum Gegenstand der Herrschaft, zum Rohmaterial erniedrigt hat“ (247). Die in der Frühgeschichte einsetzende „Entzauberung“ (11) der Welt und ihrer animistischen Belebung, die mit der Überwindung des Mythos die Übermacht der nichtdomestizierten Natur bricht und schließlich „die Beherrschung der Natur drinnen und draußen zum absoluten Lebenszweck“ erhebt (38), mündet in die Elimination des Lebendigen in der Natur. Zu einem Leitbegriff der kritischen Beschreibung wird der - auch in späteren Schriften Adornos grundlegende Begriff der Mimesis. Er steht für eine Nähe zur Natur, die über die aristotelische Nachahmung der Physis durch die Techne hinausgeht und das Subjekt in seiner ursprünglichen Lebendigkeit involviert und am Natürlichen partizipieren lässt eine Partizipation, die im instrumentellen Naturverhältnis verfemt und durch die Tendenz zum schlechten Naturalismus abgelöst wird. Aus der Mimesis ans Lebendige wird die „Mimesis ans Tote“ (64), der dem Todestriebs verschwisterte „mimétisme“ von Caillois (241) bzw. die „Mimikry mit dem Anorganischen“, die noch die gestählten Körper wie „präparierte Leichen“ erscheinen lässt (AGS 4, 64).

Es entspricht einer psychoanalytischen Perspektive, dass das gewaltsam Unterdrückte sich nicht zur Gänze austreiben lässt. Wie das Verdrängte nach Freud „nachdrängt“, so bleibt das evolutionär Unterdrückte als Tendenz und Versuchung virulent, muss der mimetische Impuls gewaltsam niedergehalten werden. Zentral ist diese Figur nicht zuletzt für die Diagnose des Antisemitismus, die in der Dialektik der Aufklärung nicht religionstheoretisch oder politisch, sondern anthropologisch ansetzt und die Nähe zur Natur als Gegenstand einer Sehnsucht erkennt, die auf den Anderen projiziert und in ihm bekämpft wird, wobei der „mimetischen Verlockung“ (193) noch in der verhöhnenden Aggression nachge- 
geben, die unterdrückte Regung auf dem Umweg der Projektion zugelassen wird (196). Die zweifache, nach innen und außen gewendete Repression demonstriert zugleich mit der tiefliegenden Ambivalenz des Naturverhältnisses die Macht der Zugehörigkeit wie der gegen sie gekehrten Abwehr. Sie mündet in einer Naturzerstörung, die in Kulturindustrie, technischer Weltbeherrschung und verdinglichendem Umgang mit sich unterschiedliche Facetten einer abgründigen Entfremdung entfaltet. Gegen sie bringt die Dialektik der Aufklärung Spuren eines versöhnten Naturbezugs in den Blick, teils in Formen und Residuen einer nicht zerstörten Mimesis, teils in jenem „Eingedenken der Natur im Subjekt“ (47), das geradezu zur Chiffre des Zugangs zum Verschütteten und Anderen, zum Kern einer Überwindung der Logik der Macht wird. Die Figur des Eingedenkens findet im Horizont der Fragen nach dem Ursprung und der Erinnerung ihre weitere Explikation.

\subsection{Ambivalenz des Ursprungs - Sehnsucht, Emanzipation, Regression}

In Auseinandersetzung mit Trieb und Natur vollzieht sich der Prozess menschlicher Selbstwerdung. Sie realisiert sich wesentlich als ein Prozess der Ablösung, der sich gegen den Ursprung wendet. Diese Gegenwendung zeigt sich als eine grundlegend ambivalente, in welcher der eigene Grund sowohl verbleibende Basis und Objekt des Strebens wie Gegenstand der Verfemung und destruktiven Unterdrückung wird. Generell ist die Suche nach dem Ursprung in der Ideengeschichte im spannungsvollen Spektrum zwischen Ursprungssehnsucht, Ursprungsverhaftung, Ursprungsverlust und Ursprungskritik zum Thema geworden (vgl. Angehrn 2007). Gilt die Suche nach dem Grund und Ersten den einen als Gewähr der Wahrheit und sicheren Orientierung, so den anderen als Indiz unfreier Verhaftung und Regression. Einem Grund entspringen kann das emanzipative Freiwerden wie das Fundiertsein in der Herkunft meinen. Diesen zwiespältigen Ursprungsbezug macht die Dialektik der Aufklärung im Werden des Individuums wie in der Urgeschichte des Subjekts zum Thema.

Es sei Aufgabe der Philosophie, meint Horkheimer 1945 in einer Vorlesung an der Columbia University, „die Erinnerung an die Mimesis der Kindheit wachzurufen, die durch die spätere Sozialisation verdunkelt worden“ ist (Jay 1976, 316). Ähnlich wendet sich Adorno 1946 gegen die revisionistische Relativierung der „zentralen Rolle der Kindheitserinnerungen“ als Kern der psychoanalytischen Theorie (AGS 8, 23). Dabei ist die Bedeutung der Kindheit nicht nur eine genealogische, sie liegt nicht allein in der Relevanz der ersten Entwicklungsphase und 
in den lebensgeschichtlichen Auswirkungen früher Entbehrung, Verletzung oder Disziplinierung. Sie liegt ebenso in der Nähe zur ursprünglichen Einheit und Geborgenheit, darin, dass Kindheit zum Ort der Sehnsucht nach Heimat und Versöhnung, der Erinnerung an das ursprüngliche Glück wird. Als Gegeninstanz zum abstrakten Gehäuse der fungiblen Gesellschaft dient das Ahnen und Wünschen des Kindes, das Adorno anschaulich mit dem Nachhall von Namen im kindlichen Erleben - in signifikant entgegengesetzter Prägung - verbindet: als Erinnerung an die Faszination, die vom Niedrigen, durch die Zivilisation Verdrängten ausging und die das Kind ,aus den Worten Luderbach und Schweinstiege ansprang“ (AGS 6, 358-359), wie als Erinnerung an das Glück, „das Namen von Dörfern verheißen wie Otterbach, Watterbach, Reuenthal, Monbrunn“ (AGS 6, 366). Die Odyssee, die das Schicksal der Genese des Selbst vor Augen führt, ist wesentlich um Motive der Sehnsucht nach Rückkehr, nach dem verlorenen Urzustand, nach Heimat angelegt (85). Dabei verkörpert die Episode der Lotophagen den abgründigen Zwiespalt des Einsseins, den Segen und Fluch des Vergessens, welches das Schlaraffenland, das Aufgehen im Unmittelbaren, aber auch die Absage an die Rückkehr bedeutet (70-71). Die Einheit und das Aufgehen im Ganzen ist sowohl utopische Erfüllung wie drohende Verschlingung. Die beschädigte Existenz bleibt in ungelöster Spannung zwischen den Fluchtpunkten der wiedererlangten Integrität und der regressiven Auflösung, die Selbstwerdung der Gattung wie des Individuums verbleibt in der Antinomie von Emanzipation und Regression. Gewaltsam wird die Lockung der Natur, die Sehnsucht nach dem Ursprung von denen bekämpft, die ihr zu verfallen drohen.

\subsection{Vergessen, Wiederholen, Erinnern}

In genuiner Verschränkung mit psychoanalytischen Konstellationen entfaltet die Dialektik der Aufklärung den zwiespältigen Ursprungsbezug in der Dialektik von Erinnern und Vergessen. Den hohen Wert des Erinnerns, der der Analyse vor Augen steht und der sich im individuellen Lebenslauf bekräftigt, gilt gleichermaßen für den geschichtlichen Vergangenheitsbezug. In Frage steht eine Erinnerung jenseits des positivistischen Berichts oder der Mnemotechnik des Gedächtnisses, eine Erinnerung, der es „nicht um die Konservierung der Vergangenheit, sondern um die Einlösung der vergangenen Hoffnung“ zu tun ist (5). Erinnerung geht nicht auf in der Reproduktion der Fakten, sondern meint ebenso ein Festhalten am Potential, an der Forderung und am Versprechen des Lebens, das in die Kindheit scheint und den Gang des Lebens trägt. Gerade wenn reale Geschichte sich als „Grauen“ erweist, ist jenes „erste Aufleuchten von Vernunft“ bedeutsam, das im Ausdruckstrieb der Kreatur sich meldet „und im erinnernden 
Denken des Menschen widerscheint“ (236-237). Erinnerung, die in abgründiger Einheit Leidenserinnerung und Erinnerung eines ursprünglichen Glücksversprechens ist, wird der Kritischen Gesellschaftstheorie zur subversiven Kraft. Deshalb ist Erinnerung in der etablierten Ordnung verpönt, wird sie von ihr bekämpft. Die Besinnung auf den Ursprung, die Erinnerung der Natur ist der herrschenden Praxis „gefährlich“ (271), das Vergangene gilt den gleichgeschalteten Individuen als irrational und suspekt, bringt sie „in Wut“ (226). Die Verdrängung der Geschichte findet ihr Echo in der Abneigung gegen die Pflege des Gedächtnisses in der revisionistischen Psychoanalyse: „La recherche du temps perdu est du temps perdu“ wird gewissermaßen zum Schlagwort einer Marginalisierung der Erinnerung, die „dem herrschenden Geist verschworen“ ist und mit der Abschaffung der Individualität paktiert (AGS 8, 34).

Einen zentralen Stellenwert in der entfremdeten Existenz wie der sozialen Realität besitzen Formen der entäußerten, nicht-gelingenden Erinnerung. Für die Psychoanalyse sind sie unter den Titeln der Verdrängung und der Wiederholung thematisch geworden: einerseits im Modus jenes zwanghaften Verbannens aus dem Bewusstsein, das doch kein befriedetes Vergessen, kein erlösendes Freiwerden - einschließlich der Trauer und des Abschieds - ermöglicht, sondern Vergangenheit als unerkannte, unverarbeitete mit sich schleppt und in Symptomen und Ersatzhandlungen sich manifestieren lässt, anderseits in der obstinaten Wiederkehr des Gleichen, die sowohl die neurotischen Zwangshandlung wie den Kreislauf der gesellschaftlichen Mechanismen strukturiert. Das urgeschichtliche Muster dieser in der Immergleichheit sich zuwiderlaufenden Entwicklung sieht die Dialektik der Aufklärung im mythischen Bann, der Unentrinnbarkeit der zyklischen Zeit, die sich ebenso in der Permanenz der Versagung im Kulturbetrieb auswirkt (17-23, 142-158). Solche Wiederholung ist selbst eine Form, eine Abart des nicht als solchen erkannten Erinnerns, die zugleich die schlechte Herrschaft des Vergangenen perpetuiert und die Offenheit der Gegenwart und Zukunft unterbindet. Eine wahre, befreiende und versöhnende Erinnerungskultur, wie sie Freud (1914) in der Schrift „Erinnern, Wiederholen, Durcharbeiten“ zur Sprache bringt, verlangt die Überführung der unbewusst-zwanghaften Wiederkehr des Vergangenen in dessen reflektierte Aneignung in einem bewussten Leben.

\subsection{Leiden und Glückserinnerung}

Entscheidend ist in alledem, dass die Frage von Erinnern und Vergessen nicht als kognitive Fähigkeit und Technik der Memoria, sondern als lebensweltlicher Vergangenheitsbezug interessiert, der wesentlich durch dessen affektiv-normative Prägung bestimmt wird. Er kommt ebenso wohl als ursprüngliche Glückserin- 
nerung wie als Leidenserinnerung zum Tragen. Beide Ausrichtungen sind für die individuelle Biographie wie die kritische Geschichtsphilosophie von Belang. Gerade die negativistische Färbung der Erinnerung ist Adorno zufolge für Freud selbst ein Motiv für deren konzeptuelle Zentralität: „Was Freud eigentlich dazu veranlaßt, einzelnen Vorgängen in der Kindheit besonderes Gewicht beizumessen, ist, obzwar unausdrücklich, der Begriff der Beschädigung“(AGS 8, 24). Gleichwohl ist es wichtig, an beiden Ausrichtungen als Angelpunkten des Selbstseins wie der Geschichte festzuhalten.

Die Rettung einer utopischen Glücksidee gegen das „verordnete Glück“ im sozialen Amusement des Kulturbetriebs (AGS 4, 67) ist Vorbedingung für die Befreiung des Individuums aus der Fessel sozialer Uniformität. Es geht zum einen um eine Rehabilitierung der von der Zivilisation verschmähten, von der psychoanalytischen Lehre aufgewerteten basalen Lust, die gewissermaßen als Statthalterin einer nicht disziplinierten sinnlichen Präsenz dient, zum anderen um das Festhalten an Bildern einer emphatischen Erfüllung, wie sie der tiefsten Sehnsucht entspringen und ,in der Erinnerung des fernsten und ältesten Glücks“ aufblitzen (71). Ausführlich beschreibt die Dialektik der Aufklärung die Eindämmung und Pervertierung dieses ursprünglichen Begehrens in der gesellschaftlich akzeptierten, ja, verordneten Genuss- und Spaßkultur, in welcher ein authentisches Glücksverlangen nicht nur beschnitten, sondern in sein Gegenteil verkehrt wird. Hatte Freud die Etablierung der Kultur noch über die verwandelnde Sublimierung der Libido erklärt, so weicht die erhöhende Sublimierung im heutigen Kulturbetrieb der nackten Repression, die im Zeichen der Versagung um eben jenes betrügt, das sie fortwährend verspricht (148-149). Indem sie „als Paradies denselben Alltag“ wieder anbietet, ersetzt sie den transzendierenden Überstieg durch die Verkettung des Immergleichen. Vergnügen wird zum „Betrug am Glück“ (149). Subversiv ist in einem solchen System die Kritik am falschen Glück ebenso wie die Erinnerung an das wahre, die beide verfemt, untersagt und unterdrückt werden.

Ineins damit aber trifft das Verbot die Erinnerung des Leidens. Die ideologische Befangenheit durchbrechen hieße die Gewalt, das Unrecht und das Leiden erkennen, welche die Signatur der Welt ausmachen. In den der Psychoanalyse gewidmeten Aphorismen der Minima Moralia spitzt Adorno diesen Gedanken zum Äußersten zu: „Es gehört zum Mechanismus der Herrschaft, die Erkenntnis des Leidens, das sie produziert, zu verbieten, und ein gerader Weg führt vom Evangelium der Lebensfreude zur Errichtung von Menschenschlachthäusern so weit hinten in Polen, daß jeder der eigenen Volksgenossen sich einreden kann, er höre die Schmerzensschreie nicht“ (AGS 4, 68). Dagegen müsste eine kritische, „kathartische“ Betrachtung darauf zielen, „die Menschen zum Bewußtsein des Unglücks, des allgemeinen und des davon unablösbaren eigenen zu bringen“ (AGS 4, 68) und die im Funktionsgefüge von Arbeit und Vergnügen sich durchsetzende 
Stummheit und Unsichtbarkeit des Leidens $\mathrm{zu}$ durchbrechen. Verlangt ist eine Macht der Kritik, die zuletzt von der Kraft der Negation, des Neinsagens nicht ablösbar ist. Abwehr und Widerstand sind nicht nur psychische Funktionen im Dienste des realitätsgerechten Ich, sondern desgleichen Kräfte des Individuums gegen die herrschende Realität, zutiefst gegen Leiden und Unrecht. Der Kritikansatz der Dialektik der Aufklärung, der in der Analyse der Kulturindustrie wie in der Auseinandersetzung mit dem Antisemitismus zum Tragen kommt, ist der fundamental negativistischen Anlage der Negativen Dialektik verwandt: das Wahre nur aus der Negation des Falschen, im unversöhnlichen Widerstand und der „unbeirrten Negation“ (AGS 6, 162) des Nichtseinsollenden zu gewinnen. Dieser Gedanke, den die Negative Dialektik in den berühmten Satz fasst: „Das Bedürfnis, Leiden beredt werden zu lassen, ist Bedingung aller Wahrheit“ (AGS 6, 29), steht auch über den Ausführungen der Dialektik der Aufklärung. Wenn die reale Geschichte nicht Fortschritt zur Humanität, sondern im Gegenteil „das Grauen ist, so ist Denken in Wahrheit ein negatives Element“ (236). Gegen das Verbleiben im Negativen wehren sich der Ausdrucksdrang des Lebens, der den Schrei der Empörung artikuliert, wie das Ethos der Kritik und das Ideal wahren Erkennens. Freud hat die praktische Abwehr als Fundament allen Negierens - die Verneinung als „intellektuellen Ersatz der Verdrängung“ (Freud 1925, 12) - und damit allen Sprechens behandelt. Die Fundamentalität der Negativitätserfahrung und -kritik durchzieht analog das Theoriegerüst der Dialektik der Aufklärung. Die Verhinderung des Negierens und Widerstehens gehört selbst zur Herrschaftstechnik der Repression (150). Die Elimination der Negation aus der Sprache bildet einen Kern jener von Marcuse gegeißelten Eindimensionalität, in welcher das Denken seinen kritischen Stachel verliert und zum Instrument der Anpassung wird (Marcuse 1967, 139-158).

Erneut im Einspruch gegen die harmonisierende Lehre von Horney und Fromm und die Tendenz zur nivellierend-angleichenden Therapie insistiert Adorno auf dem von Freud bedachten Leidensfaktor im Leben des Einzelnen und in der Dynamik der Psychoanalyse. Es gilt wohl das Individuum „als ein Absolutes zu nehmen“, dessen einheitlicher Charakter jedoch eher einem „System von Narben“ gleicht, „die nur unter Leiden, und nie ganz integriert werden“, und das „nur durch Leiden, Lebensnot“ an die gesellschaftliche Totalität gebunden ist (AGS 8, 24, 35). Wenn Psychoanalyse nicht nur eine Theorie über den Aufbau des Selbst, sondern auch ein verstehender Umgang mit Negativität und Leiden ist, so ist auch dies eine Spur, die in gleicher Weise die Nachzeichnung der Urgeschichte des Menschen in der Dialektik der Aufklärung durchzieht. 


\subsection{Der Zwiespalt der Psychoanalyse}

Die Spur Freuds in der Dialektik der Aufklärung ist weder einheitlich noch eindeutig. Uneinheitlich, teils zwiespältig erscheinen sowohl die Lehre Freuds wie die psychoanalytische Strömung und die Stellungnahme der Kritischen Theorie zur Psychoanalyse. Teils geht es dabei um konzeptuelle Veränderungen im Laufe der freudschen Theoriebildung, teils um strukturelle Antinomien in seinem Konzept, teils um Divergenzen zwischen ihm und anderen theoretischen und therapeutischen Richtungen, teils um Verschiebungen innerhalb der Positionierung Horkheimers und Adornos, aber auch anderer Angehöriger der Frankfurter Schule in ihrem Verhältnis zur Psychoanalyse. Im Folgenden seien unter diesen vielfältigen Aspekten abschließend nur zwei festgehalten: die Kritik an bestimmten Richtungen und Anwendungen der Psychoanalyse und die Auseinandersetzung mit internen Spannungen in Freuds eigenem Konzept.

Schon genannt wurde die Distanzierung gegenüber revisionistischen Aufweichungen, welche die Stringenz der Sexual- und Triebtheorie der Freudschen Theorie suspendieren. Nach anderer Richtung gilt die Kritik der auch in psychoanalytischen Strömungen auszumachenden objektivierenden Tendenz einer Psychologie, welche den Menschen als Material untersucht, in seine Fähigkeiten und Triebe zerlegt und die Analyse in den Dienst einer Technik der Seelenbeherrschung stellt (AGS 4, 69). Die kulturkritisch pointierteste Kritik zielt auf die der Psychoanalyse innewohnende Tendenz zur Normalisierung und Anpassung, die zum Teil mit der Dominanz der therapeutischen Ausrichtung der Psychoanalyse einhergeht. Als ideologieverdächtig gilt die therapeutische Orientierung, welche die sozial induzierten Pathologien als behebbar unterstellt und in der Fokussierung auf das Seelenleben des Einzelnen die „absolute Vorherrschaft der Ökonomie“ ignoriert, welche die Fassade der Normalität über die Verstümmelung der Individuen durchsetzt und eine Gesundheit dekretiert, der selbst „die Flucht in die Krankheit abgeschnitten“ ist; ungeschmälerter Polemik begegnet eine Psychoanalyse, die den herrschenden Verhältnissen „längst den Treueid geleistet“ und sich selbst „zu einem Stück Hygiene“ gemacht hat (AGS 4, 63-64). Die Tiefenpsychologie, die „mit Hilfe des Films, der Seifenopern und der Horney [...] in die letzten Löcher dringt“, macht sich zum Hilfsmittel der organisierten Kultur, indem sie die Triebkonflikte nicht heilt, doch in domestizierbare Mechanismen integriert und als Bestandstück in das genormte Leben „hineinmontiert“ (AGS 4, 71). Sie hat teil am Illusionären einer individuumszentrierten Triebökonomie, die „,in der Ära der großen Konzerne und Weltkriege“ längst „psychologisch expropriiert“ und „rationeller von der Gesellschaft selbst betrieben“ wird (213). Die Abschaffung des 
Individuums ist sowohl indirekte Folge der Bemühung um die Psyche des Einzelnen wie sie ihr vorausgeht und ihre Falschheit bedingt.

Wenn solche Vorbehalte weniger Freud selbst als andere Repräsentanten, bestimmte Praktiken und populärwissenschaftliche Adaptationen der Psychoanalyse treffen, so distanziert sich die Dialektik der Aufklärung doch auch von Freuds eigener Sichtweise. Sie tut dies im zentralen Punkt der Absage an den Fortschrittsglauben der Aufklärung, der etwa in Freuds kulturkritischen Schriften die Illusionszerstörung der Religionskritik mit der Emanzipation des Menschen verknüpft (Freud 1927; vgl. Lohmann 2006, 378). Gerade in der Kernaussage einer in sich rückläufigen Aufklärung stellt sich die Kritische Theorie in ein gespanntes Verhältnis zur leitenden Intention der Psychoanalyse. Allerdings nimmt sie dabei auch ein internes Spannungsverhältnis, einen Zwiespalt innerhalb der Freudschen Lehre wahr, die auf der Fundamentalität des „unbewußten Triebgrunds“ im Handeln und Erleben der Subjekte beharrt und gleichzeitig an der „bürgerlichen Verachtung des Triebs“ teilhat und darin der gesellschaftlichen Rationalisierung zuarbeitet: „Freuds unaufgeklärte Aufklärung spielt der bürgerlichen Desillusion in die Hände. Als später Feind der Heuchelei steht er zweideutig zwischen dem Willen zur hüllenlosen Emanzipation des Unterdrückten und der Apologie hüllenloser Unterdrückung“ (AGS 4, 65-66). Der Zwiespalt ist einer der in sich gegenläufigen, von Regression bedrohten Aufklärung, an welcher „die Psychoanalyse als ein Stück Aufklärung teilhat“ (AGS 8, 30). Inhaltlich geht es um den strukturellen Zwiespalt in der Auffassung des Subjekts, zwischen Lust- und Realitätsprinzip, aber auch zwischen individuellem Selbst und sozialer Prägung, psychologischer und soziologischer Analyse, worin einerseits die Psychoanalyse sich in den Dienst einer Befreiung des Selbst aus innerem wie äußerem Zwang stellt, andererseits das Subjekt als bloßer „Schauplatz“ des Sozialen gilt und die Psychoanalyse als hilfloser Versuch erscheint, „das bereits nicht mehr vorhandene Individuum zu retten“ (HGS 12, 440), ja, in ihrer desillusionierten Version die Persönlichkeit geradezu als „Lebenslüge“ einzieht und die Inkommensurabilität des Einzelnen sabotiert (AGS 4, 70 -72).

Indes entspricht dieser zweifache Blick auf das Individuum zuletzt einem inneren Zwiespalt der Subjektwerdung, wie er auch der existenzphilosophischen Reflexion gegenwärtig ist und in Freuds Schriften teils einseitig von der einen, teils der anderen Seite angegangen wird. Der Selbstbezug des Einzelnen ist darin ebenso irreduzibel wie seine Situierung im Historisch-Sozialen unhintergehbar ist. Gerade die Intransigenz des Festhaltens an beiden Polen des Menschseins wird für die Theorie zum Index der Wahrheit. Es ist ein Beipflichten zu dieser Unnachgiebigkeit, wenn Adorno das bekannte Diktum formuliert, an der Psychoanalyse sei „nichts wahr als ihre Übertreibungen“ (AGS 4, 54), oder schreibt, „Freud hatte recht, wo er unrecht hatte“ (AGS 8, 35) - wie die Dialektik der Aufklärung auch mit 
Bezug auf den provozierenden Amoralismus der „dunklen Schriftsteller des Bürgertums“ feststellt: „Aber nur die Übertreibung ist wahr“ (126). In der Stellungnahme zur Psychoanalyse dominiert zumal bei Adorno jene Unversöhntheit der Gegensätze, welche die Negative Dialektik gegen den spekulativen Vermittlungsanspruch der Hegelschen Dialektik zum Programm erhebt. Eben darin erkennt er die Wahrheit der psychoanalytischen Theorie: „Die Größe Freuds besteht wie die aller radikalen bürgerlichen Denker darin, daß er solche Widersprüche unaufgelöst stehen läßt und es verschmäht, systematische Harmonie zu prätendieren, wo die Sache selber in sich zerrissen ist.“ (AGS 8, 40) Für Kritische Gesellschaftstheorie geht es darin nicht nur um das Schicksal des Psychischen oder die antinomische Verfassung der Existenz, sondern um die objektive Unvernunft und Unversöhntheit geschichtlicher Realität.

\section{Literatur}

Angehrn, Emil. 2007. Die Frage nach dem Ursprung. Philosophie zwischen Ursprungsdenken und Ursprungskritik, München

Bonß, Wolfgang. 1982. Psychoanalyse als Wissenschaft und Kritik. Zur Freudrezeption der Kritischen Theorie, in: Bonß, Wolfgang/Honneth, Axel (Hrsg.), Sozialforschung als Kritik. Zum sozialwissenschaftlichen Potential der Kritischen Theorie, Frankfurt a.M., 367-425

Dahmer, Helmut. 2012. Die unnatürliche Wissenschaft. Soziologische Freud-Lektüren, Münster Freud, Sigmund. 1914. Erinnern, Wiederholen und Durcharbeiten, in: Gesammelte Werke, Bd. 10, Frankfurt a.M. 1946, 126-136

Freud, Sigmund. 1925. Die Verneinung, in: Gesammelte Werke, Bd. 14, Frankfurt a.M. 1948, 11-15

Freud, Sigmund. 1927. Die Zukunft einer Illusion, in: Gesammelte Werke, Bd. 14, Frankfurt a.M. 1948, 325-380

Jay, Martin. 1976. Dialektische Phantasie. Die Geschichte der Frankfurter Schule und des Instituts für Sozialforschung 1923-1950, Frankfurt a.M.

Lohmann, Hans-Martin. 2006. Kritische Theorie, in: Freud-Handbuch, hg.v. Hans-Martin Lohmann/Joachim Pfeiffer, Stuttgart, 377-382

Marcuse, Herbert. 1967. Der eindimensionale Mensch. Studien zur Ideologie der fortgeschrittenen Industriegesellschaft, Neuwied / Berlin

Marcuse, Herbert. 1969. Triebstruktur und Gesellschaft. Ein philosophischer Beitrag zu Sigmund Freud, Frankfurt a.M.

Rantis, Konstantinos. 2001. Psychoanalyse und „Dialektik der Aufklärung“, Lüneburg 